\title{
Syncope Due to Intracavitary Left Ventricular Obstruction Secondary to Giant Esophageal Hiatus Hernia
}

\author{
Kazuhito Hirata*, Yuji Shimabukuro, Takanori Takahashi, Minoru Wake \\ Division of Cardiology, Okinawa Chubu Hospital, 281 Miyazato, Uruma, Okinawa, Japan \\ *Corresponding author: kheart911@yahoo.co.jp
}

\begin{abstract}
Esophageal hiatus hernia has been known to cause symptoms of congestive heart failure or syncope after meal due to compression of the heart, mainly the left atrium. However, compression of the left ventricle resulting in syncope has never been reported. Seventy-one year old woman developed syncope after taking a lunch. A chest roentgenogram showed possible mass lesion behind the heart. An echocardiogram revealed a mass lesion posterior to the left ventricle, resulting in "banana-shaped" deformity. A CT scan revealed a giant esophageal hiatus hernia which compressed the left ventricle. Cardiac catheterization revealed marked pressure gradient in the left ventricle suggestive of intracavitary obstruction after isoproterenol infusion. Left ventricular compression due to enlarged giant hiatus hernia after meal resulted in intracavitary obstruction of the left ventricle, causing syncopal episode in this particular patient. Dehydration and diastolic dysfunction may also have contributed.
\end{abstract}

Keywords: esophageal hiatus hernia, left ventricular obstruction, syncope

Cite This Article: Kazuhito Hirata, Yuji Shimabukuro, Takanori Takahashi, and Minoru Wake, "Syncope Due to Intracavitary Left Ventricular Obstruction Secondary to Giant Esophageal Hiatus Hernia." American Journal of Medical Case Reports, vol. 5, no. 4 (2017): 89-93. doi: 10.12691/ajmcr-5-4-4.

\section{Introduction}

Esophageal hiatus hernia has been known to cause symptoms of congestive heart failure or syncope after meal due to compression of the heart, mainly the left atrium. However, compression of the left ventricle resulting in syncope has never been reported.

\section{Case Presentation}

A 71-year-old woman was taken to the emergency room due to syncope. After a walk in the garden in hot weather, she had lunch. She then rested by sitting on a chair, during which she felt nauseated and developed syncopal episodes twice. She had no chest pain, dyspnea, or palpitation before syncope.

Her past medical history included hypertension, post herpetic neuralgia and gastric ulcer. Current medication included enalapril $5 \mathrm{mg}$ every day for hypertension and pregabarin for neuralgia.

On arrival, her vital signs were as follows: blood pressure of $94 / 60 \mathrm{mmHg}$, heart rate of 88 beats/minute, and respiratory rate of 24 breaths/minute, body temperature of $35.3^{\circ} \mathrm{C}$. Oxygen saturation was $96 \%$ while breathing ambient air. Jugular venous pressure was normal and she did not have edema or abnormal breath sound. Auscultation of the heart revealed grade 3 systolic ejection murmur at second right and third left interspace.
Laboratory tests were unremarkable except for mildly elevated blood urea nitrogen of $36 \mathrm{mg} / \mathrm{dl}$ (normal range: $8-21 \mathrm{mg} / \mathrm{dl}$ ); creatinine levels were normal at $0.74 \mathrm{mg} / \mathrm{dl}$ (normal range: $<0.8 \mathrm{mg} / \mathrm{dl}$ ). A chest roentgenogram showed mild cardiomegaly and double contour of the cardiac silhouette suggestive of mass lesion in the mediastinum (Figure 1 A). There was no sign of congestion. An electrocardiogram showed normal sinus rhythm and mild high voltage but without ST-T wave abnormality (Figure 1 B). A bedside echocardiogram showed a mass posterior to the left ventricle (LV), compressing LV resulting in banana-shaped deformity (Figure $2 \mathrm{~A}$ ). Mild valvular aortic stenosis (peak gradient $<20 \mathrm{mmHg}$ ) was also noted. A mass lesion located posterior to the heart turned out to be a giant hiatus hernia as confirmed by $\mathrm{CT}$ scan of the abdomen and the chest (Figure 3 A-C). There was no localized hypertrophy of LV suggesting asymmetric septal hypertrophy. Repeated echocardiography at fasting state did show the mass posterior to the LV but the size was smaller, and there was no banana-shaped deformity (Figure $2 \mathrm{~B}$ ). There was no pressure gradient in the LV outflow tract by Doppler.

To explore possible cardiac cause of syncope, cardiac catheterization and electrophysiology study were performed. A coronary angiography and electrophysiology study did not show significant abnormalities. A fluoroscopy and subsequent left ventriculography showed that the herniated stomach was located posterior to the LV, not at the atrial level (Figure 4 A B). After a spontaneous PVC, systolic arterial pressure and the pulse pressure dropped (usually increases after PVC), which is 
suggestive of the Brockenbrough phenomenon. There was 10-15 mmHg pressure gradient between the LV and aorta, suggesting mild valvular aortic stenosis, but there was no pressure gradient inside the LV at baseline (Figure 5A). After isoproterenol infusion, LV pressure increased up to $180 \mathrm{mmHg}$ to $200 \mathrm{mmHg}$ whereas aortic pressure dropped below $100 \mathrm{mmHg}$, resulting in $80 \sim 100 \mathrm{mmHg}$ pressure gradient inside the LV (Figure5B). Moreover, the shape of the aortic pressure showed systolic double peak consistent with bisferiens pulse.

Based on the history, initial bedside echocardiogram and cardiac catheterization, we concluded that the main underlying pathophysiology of syncope is external compression of the LV resulting in both impaired LV filling and intracavitary obstruction of LV. Dehydration due to a walking in a hot weather may also have contributed to syncope.
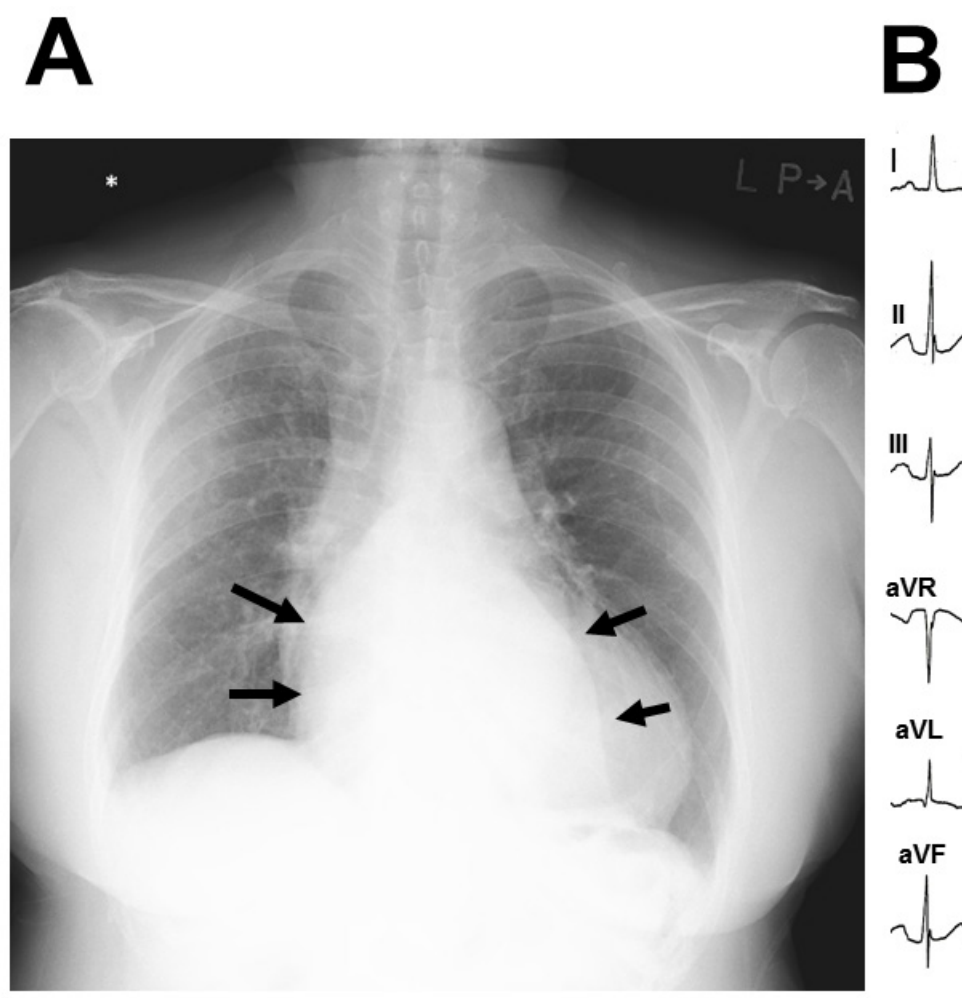

YL Dpm
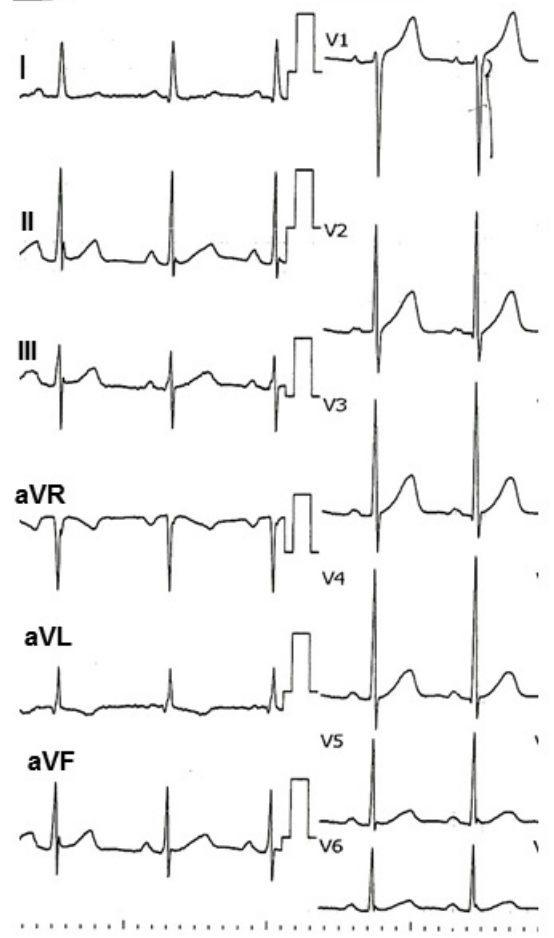

gure 1. A: A chest roentgenogram showing cardiomegaly and double contour of the cardiac silhouette. B: A twelve-lead electrocardiogram showing mild high voltage without ST-T abnormalities
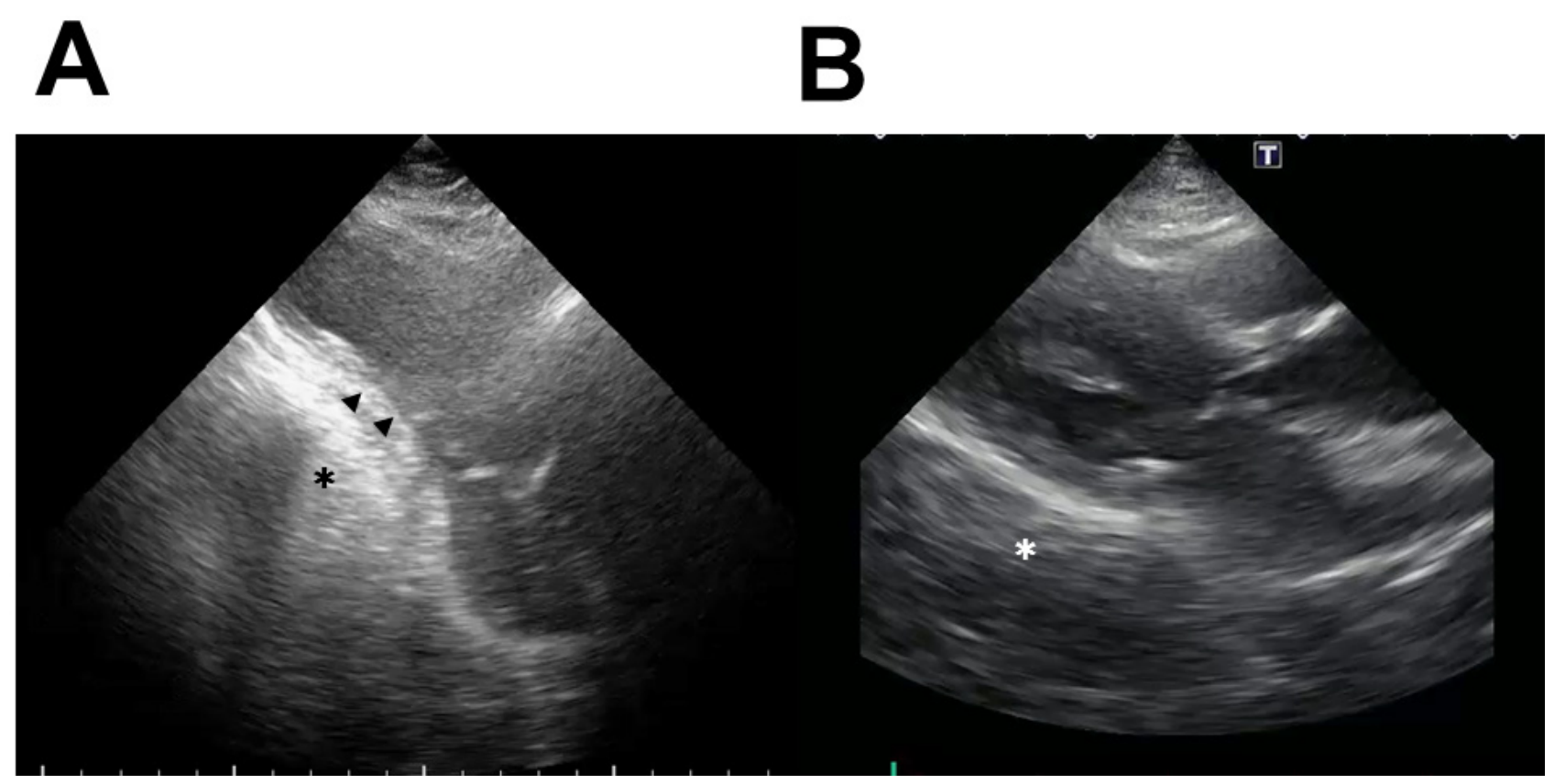

Figure 2. A: A bedside-echocardiogram at initial presentation showed compression of the left ventricle (black arrow heads) by a mass lesion (black asterisk). B: During a fasting state, the size of the mass became smaller (white asterisk) and there was no apparent compression of the left ventricle 


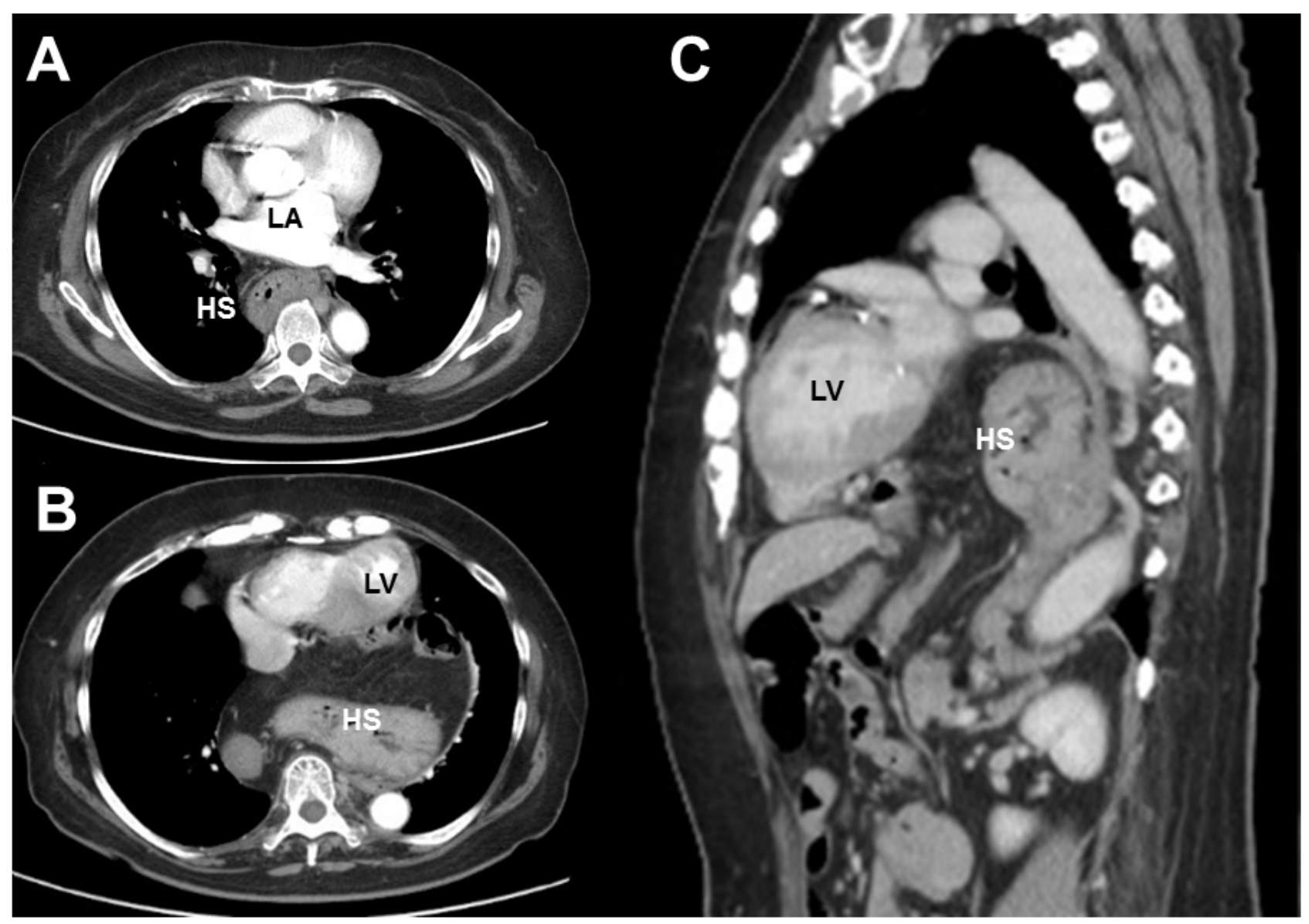

Figure 3. A-C: Computed tomography of the chest and abdomen showing giant esophageal hiatus hernia (stomach was herniated with omentum and the part of the colon) compressing the left ventricle from behind, while the left atrium was not compressed (A). LV: left ventricle, LA: left atrium, HS: herniated stomach

\section{A}
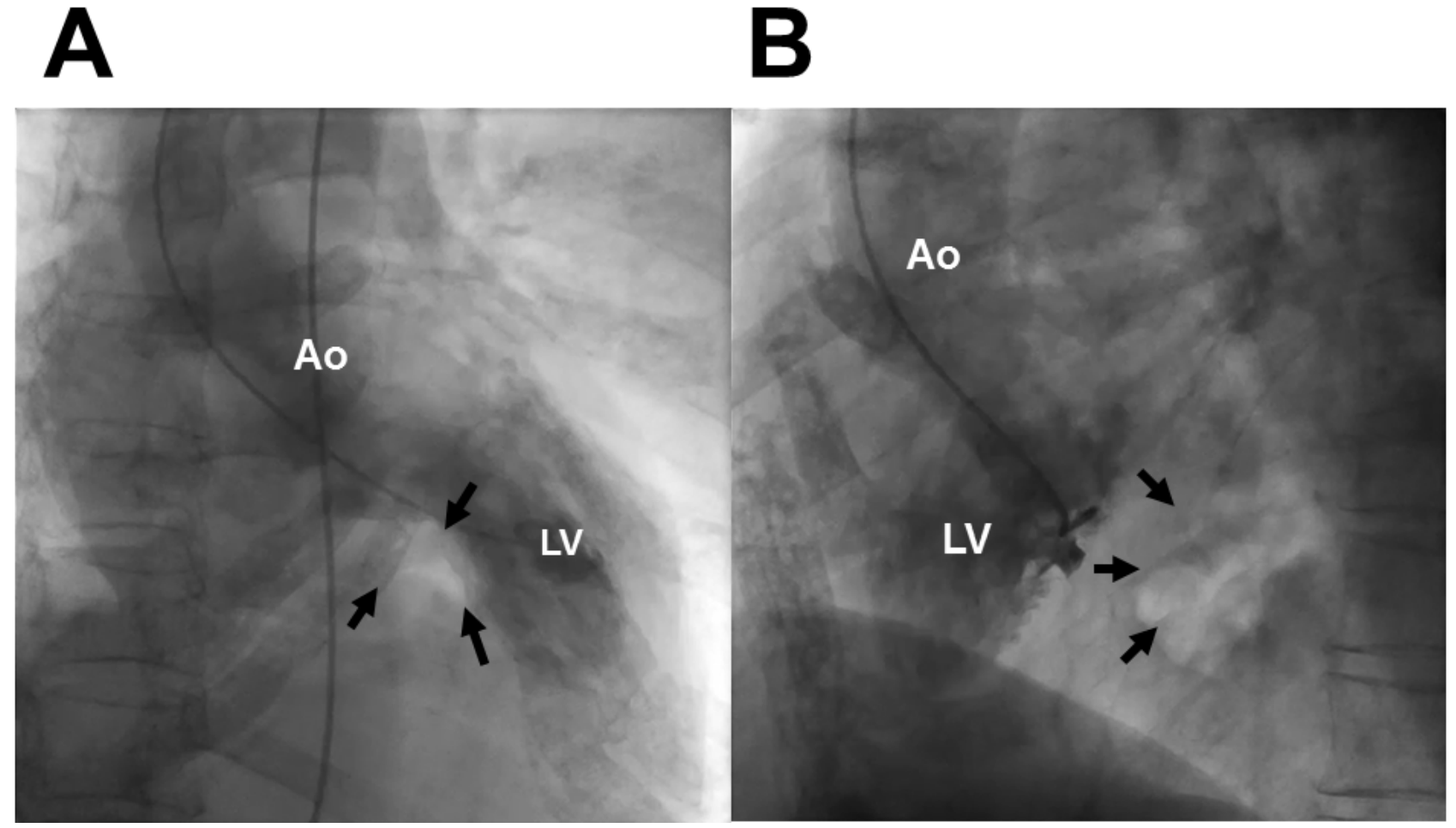

Figure 4. A and B: Left ventriculogram revealed that the gastric babble was located infero-posterior to the left ventricle. A: right anterior oblique view, B: Left anterior oblique view 


\section{A B}
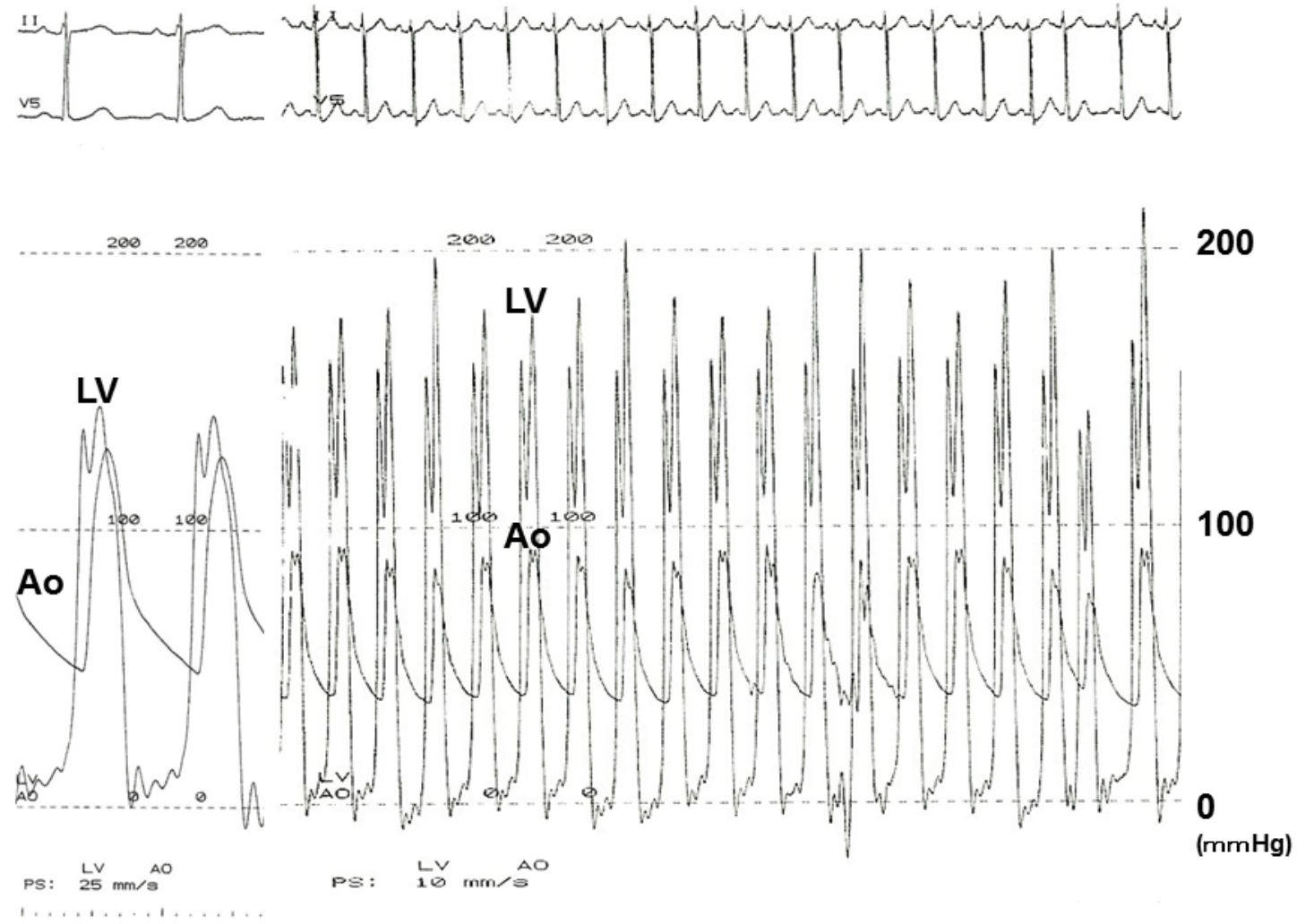

Figure 5. A: There was mild pressure gradient across the aortic valve suggestive of mild aortic stenosis. B: After isoproterenol, LV pressure at LV apex increased up to $180-200 \mathrm{mmHg}$ while arterial pressure dropped down to $80 \mathrm{mmHg}$, resulting in $80-100 \mathrm{mmHg}$ intracavitary LV pressure gradient. Note that the arterial wave form showed double peaks consistent with bisferiens pulse due to intracavitary LV obstruction

\section{Discussion}

Giant esophageal hiatus hernia can cause congestive heart failure (CHF) and syncope $[1,2,3,4]$. The mechanism for CHF includes external compression of the left atrium, resulting in increased left atrial pressure and subsequent pulmonary venous congestion $[1,2,3]$. The presence of syncope can be explained by impaired filling of LA (and subsequent impaired LV filling), resulting in decreased cardiac output. These hemodynamic changes may lead to catastrophic events [5]. Occasionally, swallowing itself causes vagally mediated syncope known as "swallowing syncope" [4].

In the present case, the left atrium was not compressed, but the LV was, as suggested by multiple imaging modalities. Although there was no resting pressure gradient in the LV at baseline, dynamic intracavitary obstruction of LV was clearly and easily reproduced by isoproterenol challenge test. We concluded that the LV is continuously compressed by the giant hernia, resulting in a banana-shaped deformity, but the degree of compression is not severe enough to cause impaired filling or intracavitary LV obstruction in the fasting state. The obstruction can be reproduced with provocative conditions such as PVC or catecholamine infusion [6]. After meals, the herniated stomach is filled with food, becomes larger, and compresses the LV resulting in impaired filling and intracavitary LV obstruction. In addition, after walking outside in hot weather (mean temperature in Okinawa on September: $27.6^{\circ} \mathrm{C}: 25.5-30.4^{\circ} \mathrm{C}$ ), the patient was in a dehydrated state as suggested by elevated BUN and normal creatinine, which was also a predisposing factor for intracavitary LV obstruction.

The possibility of latent form of hypertrophic obstructive cardiomyopathy appeared to be less likely because there was no abnormal local hypertrophy of the left ventricle such as asymmetric septal hypertrophy by echocardiography and CT scan. Neither, there were no signs of LV hypertrophy on ECG. History was not consistent with vagally mediated swallowing syncope, because syncope occurred after the lunch, not while she was swallowing. In order to prove the direct effect of the size of giant hiatus hernia on dynamic intracavitary obstruction of the LV, we should have done rapid fluid challenge test by infusing normal saline through nasogastric tube under echocardiography or during cardiac catheterization [3]. But it was not possible because she was a traveller and desired to go back to her hometown early.

\section{References}

[1] Maekawa T, Suematsu M, Shimada T, Go M, Shimada T. Unusual swallow syncope caused by huge hiatal hernia. Intern Med 2002; 41: 199-201.

[2] Naoum C, Falk GL, Ng ACC et al. Left atrial compression and the mechanism of exercise impairment in patients with large hiatal hernia. J Am Coll Cardiol. 2011;58(15):1624-1634.

[3] Nakabayashi K, Hirata T, Oka T. Left atrium compression due to oesophageal dilatation can induce acute heart failure. BMJ case rep. 2015. 
[4] Mitra S, Ludka T, Rezkalla SH et al. Swallow syncope. Clinical Medicine and Research: 9; 125-9.

[5] Neuman L, Poulton B, Ridley $\mathrm{S}$ et al. Life threatening complications of hiatus hernia. Anaesthesiology 1999; 54: 93-4.
[6] Hashimoto Y, Reid CL, Gardin JM. Left ventricular cavitary geometry and dynamic intracavitary left ventricular obstruction during dobutamine stress echocardiography. Am J Card Imaging 1996; 10: 163-9. 